\title{
Influencia de el niño oscilación sur 2016 - 2017 en los desembarques de la pesca artesanal en la region piura
}

\section{Influence of el niño oscilación sur 2016 - 2017 on artisan fishing landings in the piura region}

\author{
Juan Tume Ruiz ${ }^{1}$, Antia Rangel Vega ${ }^{2}$, Oscar Vásquez Ramos ${ }^{3}$, Percival Mendoza Ayala ${ }^{4}$
}

\section{RESUMEN}

En el presente estudio se describe la influencia que el “El Niño Oscilación del Sur” 2016 y 2017, influye en principales recursos que sustentan la pesca artesanal en la zona norte, para este estudio se tomo información de las descarga de especies desde setiembre 2016 hasta marzo 2017, para ello se analiza la relación que existe entre el ndice Costero de "El Niño", parámetro que refleja el cambio en las temperaturas superficiales del mar y que se usa para definir la presencia de El niño a nivel local y las descargas de los recursos.

Se determinó que los recursos afectados son Caballa, Cachema, Samasa, los cuales bajan sus volúmenes de descarga, a medida que el ICEN aumenta, por lo tanto, decimos que son especies afectadas por el Evento El Niño 2016 y 2017. Sin embargo, existen especies que aparecen en grandes cantidades de descarga, así tenemos al Barrilete, Chiri y Langostino, especies que pueden ser potenciales desde el punto de vista de la pesca de los recursos, y que se deben impulsar cuando hay entrada de agua dulce.

Palabras claves: El Niño, ICEN, Oscilación Sur, Pesca Artesanal, Niño costero

\section{ABSTRACT}

This study describes the influence that the "El Niño Southern Oscillation" 2016 and 2017, influences the main resources that support artisanal fishing in the north, for this study information was taken on the discharge of species from September 2016 to March 2017, for this, the relationship between The Coastal Index of "El Niño" is analyzed, a parameter that reflects the change in sea surface temperatures and is used to define the presence of El Niño at the local level and the discharges of the resources.

It was determined that the affected resources are Mackerel, Cachema, Samasa, which decrease their discharge volumes, as the ICEN increases, therefore, we say that they are species affected by the 2016 and 2017 El Niño Event. However, there are species that appear in large quantities of discharge, thus we have Skipjack tuna, long- finned butterfish and shrimp, species that can be potential from the point of view of resource fishing, and that must be promoted when there is water entry. sweet.

Keywords: El Niño Southern Oscillation, Artisanal Fishing, ICEN, Costal El Niño

1. Director del Departamento de Ingeniería Pesquera de la Universidad Nacional de Piura jtumer@unp.edu.pe

2. Docente de la Facultad de Ingeniería Pesquera de la Universidad Nacional de Piura arangelv@unp.edu.pe

3. Decano de la Facultad de Ingeniería Pesquera de la Universidad Nacional de Piura ovasquezr@unp.edu.pe

4. Egresado de la Facultad de Ingeniería Pesquera percivalmendoza@hotmail.com 


\section{INTRODUCCIÓN}

La pesca artesanal y de pequeña escala tiene un gran aporte en la sociedad en general, en términos de provisión de alimentos, empleo y divisas en los últimos tiempos, ha propiciado la realización de diversas acciones gubernamentales para tratar de incorporarla como una actividad estratégica para el desarrollo económico de los países y elevar el nivel de vida de los pescadores y sus comunidades.

La pesca artesanal abastece la mayor parte de las especies para consumo humano directo, que terminan en los principales mercados del país, especialmente en estado fresco. (Galarza et al., 2015) El Niño Oscilación del Sur" (ENOS) produce grandes cambios en el ecosistema marino y juega un importante papel en la dinámica biológica de los recursos hidrobiológicos del Océano Pacífico Sudoriental. La pesquería artesanal es una fuente importante de ingresos económicos y seguridad alimentaria que se ve fuertemente afectada por el ciclo de El Niño Oscilación Sur" (Adams \& Flores, 2016a).

El presente estudio se analiza la influencia que el "El Niño Oscilación del Sur" 2016 y 2017, tiene en los principales recursos que sustentan la pesca artesanal en la zona norte.

\section{MATERIALES Y MÉTODOS.}

Se recopiló información histórica de descargas de cada desembarcadero pesquero artesanal de Paita, Talara y Sechura. Tomando información de descargas reportadas de cada especie, por PRODUCE e IMARPE, para el Evento el Niño $2016-2017$.

En el análisis del Evento el Niño se utilizó el ICEN (Î́ndice Costero El Niño) que consiste en la media corrida de tres meses de las anomalías mensuales de la temperatura superficial del mar (TSM) en la región Niño 1+2. La fuente de datos para este índice son las TSM absolutas del producto ERSST v3b de la NOAA (EEUU) para la región Niño 1+2. Luego se relacionó el ICEN y las descargas de cada recurso hidrobiológico. Los mapas de temperatura superficial del mar, son los emitidos por el IMARPE.

Con relación a la organización y depuración de la investigación, es decir la inclusión de información que tuvo como criterio el objetivo de la revisión. El análisis de la información parte del desarrollo de las síntesis de la información que permitió identificar los tópicos que aportan al presente trabajo.

\section{RESULTADOS}

Condiciones Climáticas de temperatura superficial del mar para la zona de estudio
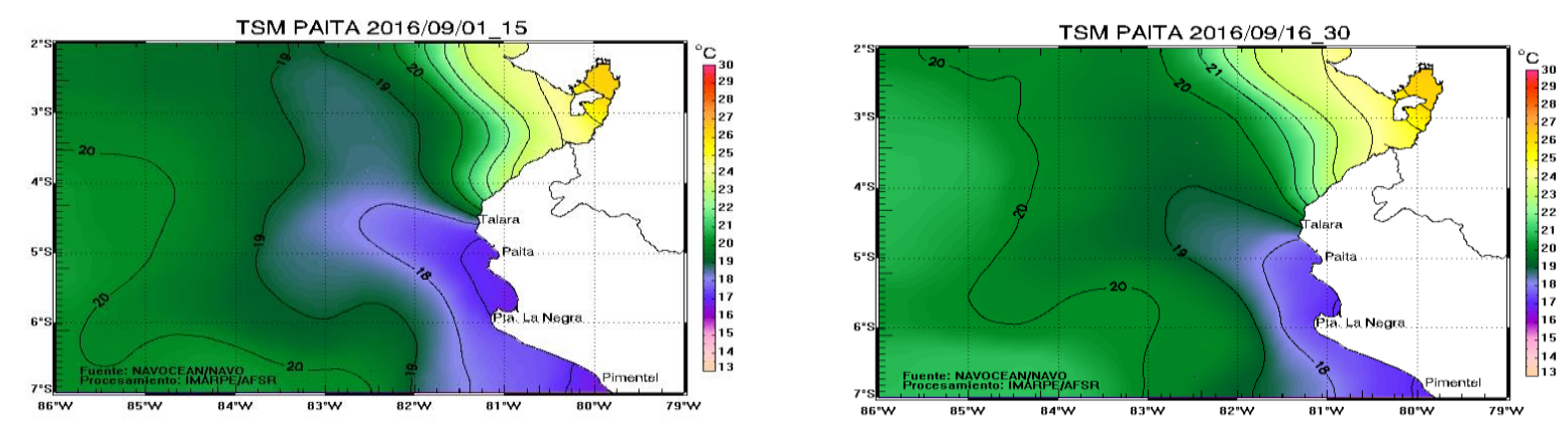

Imagen 1: Condiciones de TSM de la primera y segunda quincena de setiembre 2016 


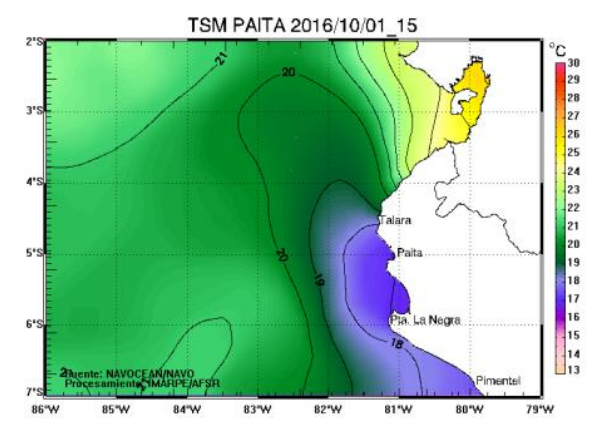

Imagen 2: Condiciones de TSM, de la primera y segunda quincena de octubre del 2016
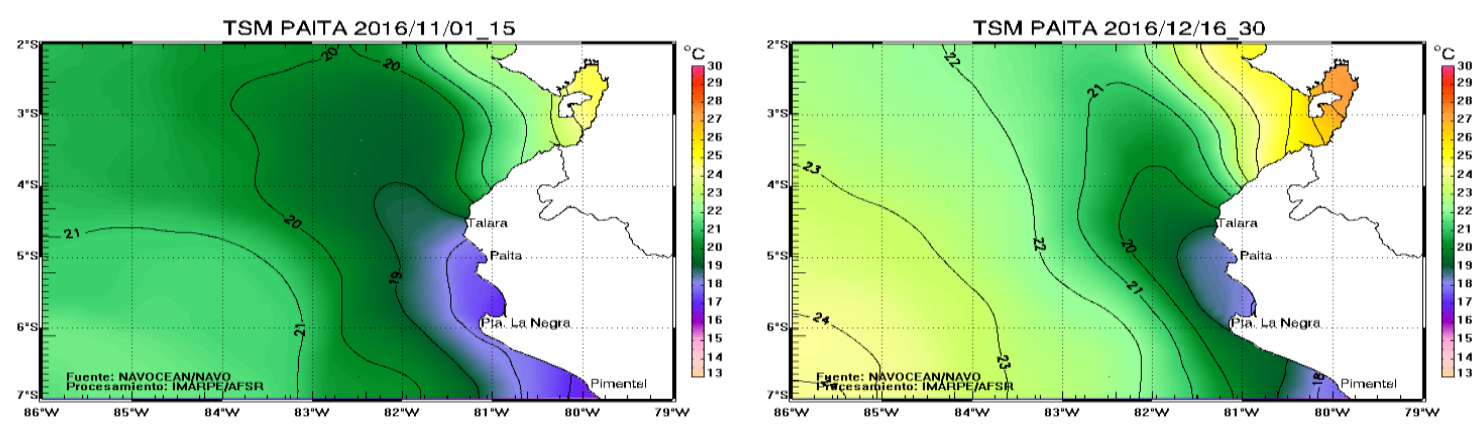

Imagen 3: Condiciones de TSM, de la primera y segunda quincena de noviembre del 2016
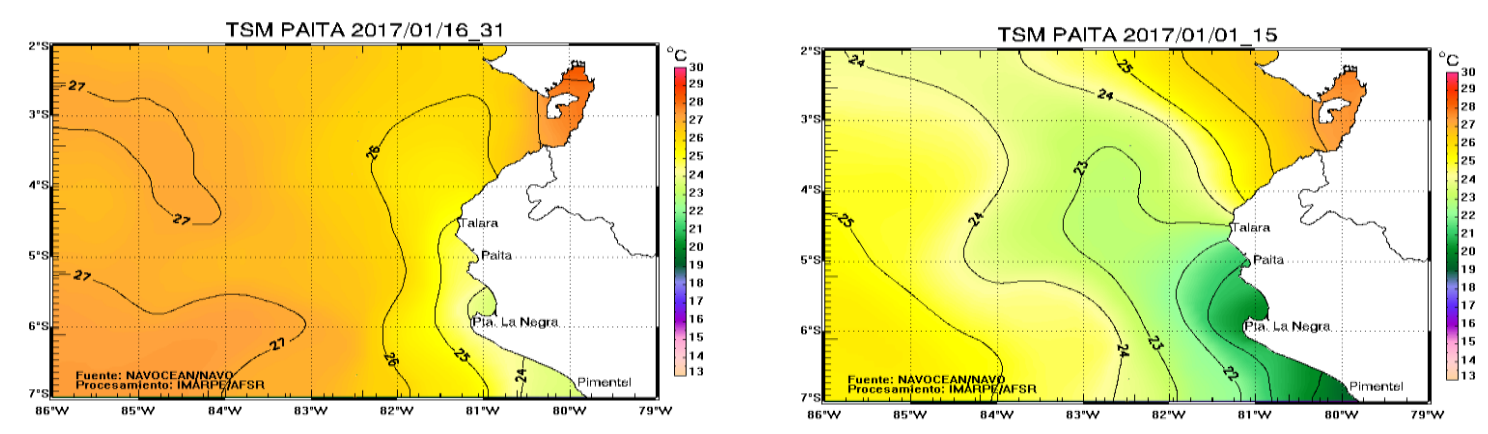

imagen 4: Condiciones de TSM, de la primea y segunda quincena de diciembre del 2016
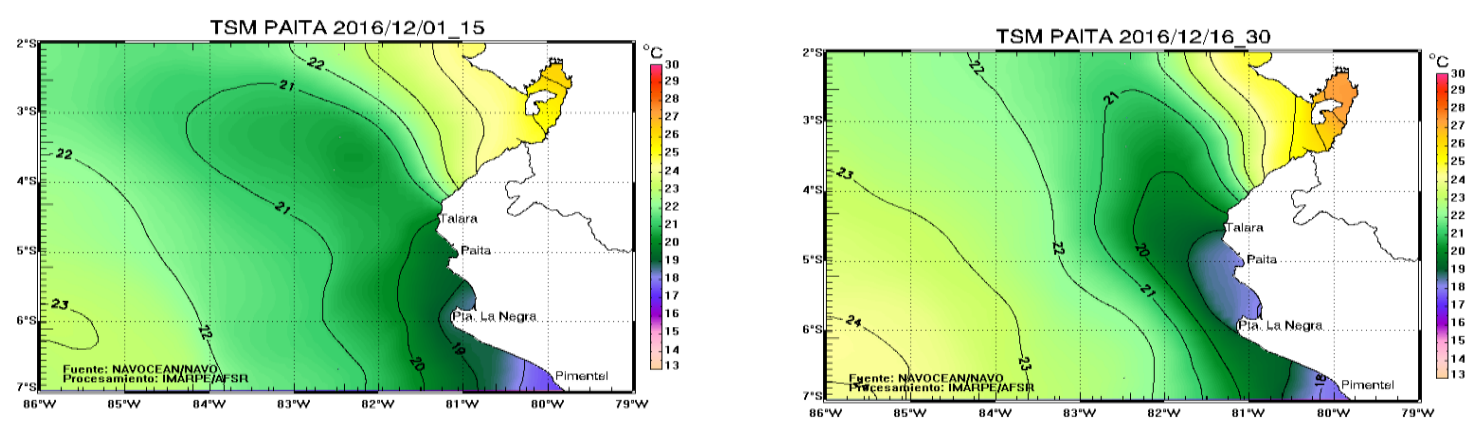

Imagen 5: Condiciones de TSM, de la primera y segunda quincena de enero del 2017 
Imagen 5: Condiciones de TSM, de la primera y segunda quincena de enero del 2017
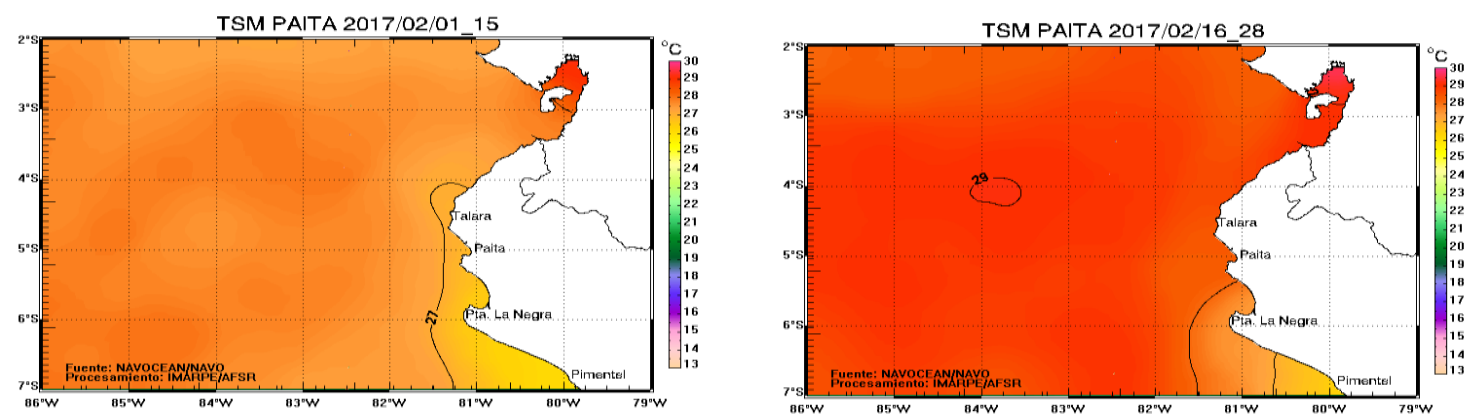

Imagen 6: Condiciones de TSM, de la primera y segunda quincena de febrero del 2017
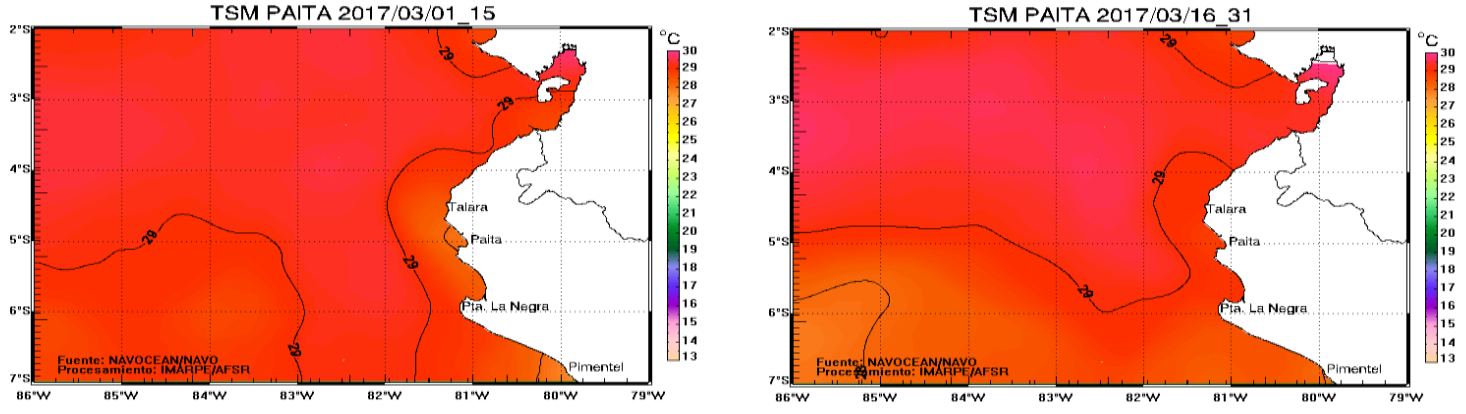

Imagen 7: Condiciones de TSM, de la primera quincena de marzo del 2017

\section{RESULTADOS}

En la imagen 1 se presenta las condiciones de temperatura superficial del mar (TSM), de la primera y segunda quincena del mes de setiembre del 2016, observamos un aumento de la primera quincena con rangos entre 18 y $20^{\circ} \mathrm{C}$, con la incursión de aguas calientes del sur Ecuatoriano, la segunda quincena el rango aumento entre $18 \mathrm{y}$ $21^{\circ} \mathrm{C}$, En octubre imagen 2, se aprecia el proceso de calentamiento, el rango es de 18 a $21^{\circ} \mathrm{C}$, pero se reduce el área de $18^{\circ} \mathrm{C}$, y se incrementa la de 20C, En Noviembre imagen 3, el área de $18^{\circ} \mathrm{C}$, sigue reduciendo, por la incursión de aguas oceánicas calientes, los rangos de temperatura siguen aumentando desde $18{ }^{\circ} \mathrm{C}$, cerca de la costa hasta $22^{\circ} \mathrm{C}$, en la parte oceánica, En la imagen 4 Se observa que sigue aumentando la TSM, en diciembre, esta vez el área de las masas de agua de $18^{\circ} \mathrm{C}$, ha desaparecido, llegando a rangos de temperatura de 20 a $23^{\circ} \mathrm{C}$ en la primera quincena y de 20 a $24^{\circ} \mathrm{C}$ en la segunda quincena.

Lo que ya es un indicio de la presencia de El niño. En enero imagen 5, observamos que las masas de agua frías han sido desplazadas por las aguas calientes amarillas y naranjas, que se acercan a la costa y son ya las responsables de las lluvias de verano en la zona norte por la presencia del fenómeno "El niño". La variación de temperatura es de 24 hasta $27^{\circ} \mathrm{C}$. En febrero imagen 6, observamos que la temperatura del mar de casi toda la zona de estudio es de $27^{\circ} \mathrm{C}$. durante la primera quincena.

La TSM siguió aumentando en la segunda quincena alcanzando $28^{\circ} \mathrm{C}$, lo que nos demuestra la presencia del fenómeno "El niño", en su con presencia de lluvias, muy fuertes. Durante el mes de marzo imagen 7 las TSM, llegaron a su valor máximo de $29^{\circ} \mathrm{C}$, y fue el mes más lluvioso que golpeó al norte del Perú, 


\section{Variación temporal del Índice Costero El Niño (ICEN)}

Imagen 8: Variación del Índice Costero de El Niño de setiembre 2016 a marzo 2017

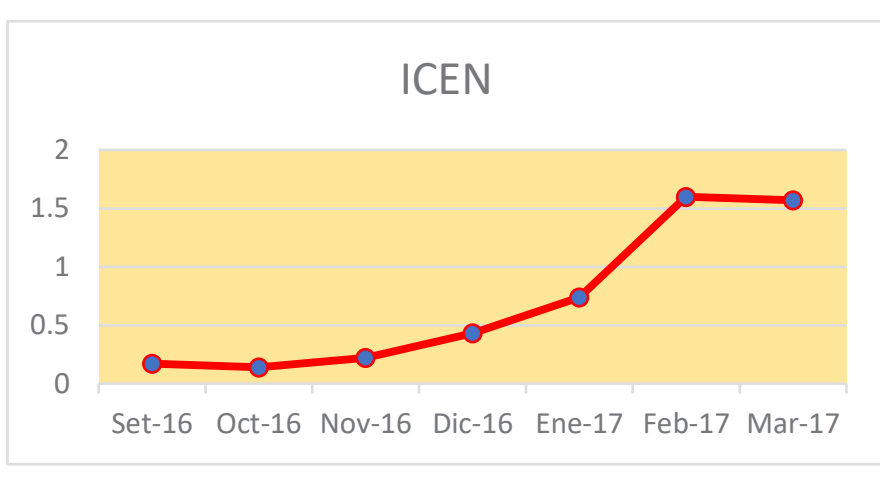

La Variación de índice Costero de El Niño, el cual tiene una tendencia a aumentar a medida que pasa el tiempo, es en diciembre donde se aprecian los incrementos con pendientes más grandes, siendo entre febrero y marzo, el mayor valor de diferencia para el ICEN, esto también indica la presencia del fenómeno El niño.

Capturas de la Pesca Artesanal en el Norte del Perú durante el niño 2016 - 2017

Tabla 1: Volúmenes de captura de las diferentes especies capturadas por la pesca artesanal

\begin{tabular}{|c|c|c|c|c|c|c|c|}
\hline \multirow[b]{2}{*}{ ESPECIES } & \multicolumn{7}{|c|}{ MESES } \\
\hline & $\begin{array}{c}\text { Set. } \\
2016\end{array}$ & $\begin{array}{c}\text { Oct. } \\
2016\end{array}$ & $\begin{array}{l}\text { Nov. } \\
2016\end{array}$ & $\begin{array}{c}\text { Dic. } \\
2016\end{array}$ & $\begin{array}{l}\text { Ene. } \\
2017\end{array}$ & $\begin{array}{l}\text { Feb. } \\
2017\end{array}$ & $\begin{array}{l}\text { Mar. } \\
2017\end{array}$ \\
\hline Pota Dosidicus gigas & 3813 & 2356 & 3711 & 1687 & 4376 & 5202 & 2825 \\
\hline Caballa Scomber japonicus peruanus & 1170 & 616 & 208 & 463 & 0 & 0 & 350 \\
\hline Cabrilla Paralabrax humeralis & 15.5 & 9.5 & 2.35 & 3.8 & 7 & 0 & 0 \\
\hline Cachema Cynocion analis & 27.3 & 9.5 & 2.55 & 3.7 & 1.3 & 1.2 & 1 \\
\hline Lisa Mugil cephalus & 6.2 & & 0 & 495 & 0 & 0 & 0 \\
\hline Samasa Anchoa nasus & & 340 & 815 & 40 & 0 & 10 & 372 \\
\hline Barrilete Katsuwonus pelamis & & & 0.5 & 1.5 & 2.65 & 104 & 80.9 \\
\hline Chiri Peprilus medius & 0.8 & 0.9 & 1.3 & 1.5 & 6.4 & 90 & 325 \\
\hline Langostino Litopenaeus vannameii & & & & 5 & 15 & 190 & 92 \\
\hline
\end{tabular}

En la Tabla 1, se presentan las descargas por especies durante setiembre 2016 y marzo 2017, observamos que la pota fue la especie más capturada en la zona norte y es una especies que fue reportada durante todo el tiempo de estudios, asimismo otras especies que se encontraron fueron la Cachema y Cabrilla, la primera especialmente con descargas en Paita y la segunda con descargas en Talara, especies que si bien fueron afectadas por presencia de aguas calientes, su captura fue mayores profundidades. Especies como el chiri, el barrilete y el langostino aparecen en los reportes a partir de diciembre y aumentan en todo el verano. 


\section{Relación entre las descargas y el ICEN}

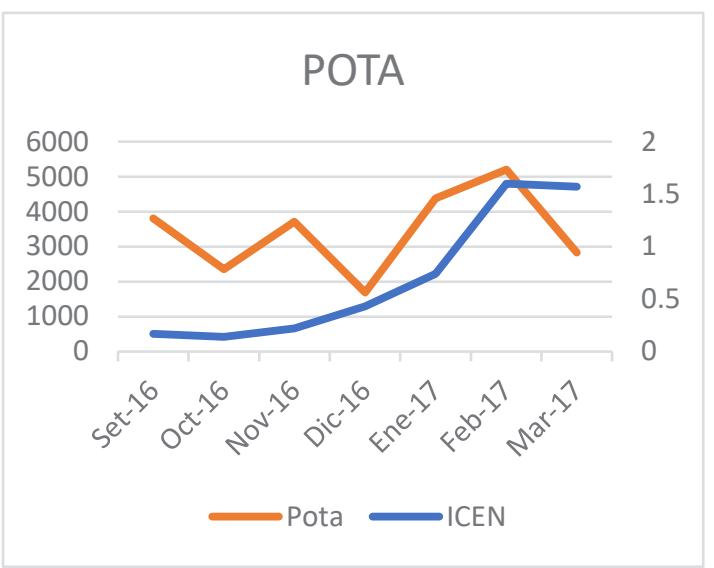

Grafico 1: Relación de las descargas de Dosidicus gigas Pota con el ICEN, de setiembre 2016 a marzo 2017

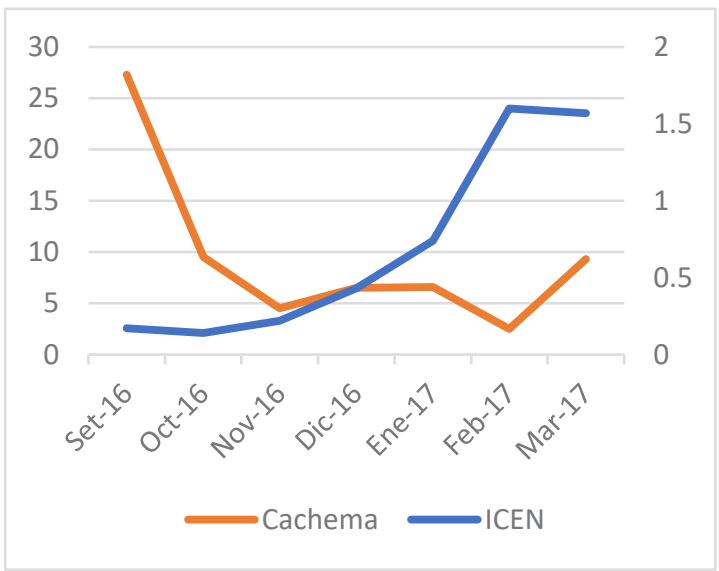

Gráfico 3: Relación de las descargas de Cachema Cynocion analis con el ICEN, de setiembre 2016 a marzo 2017

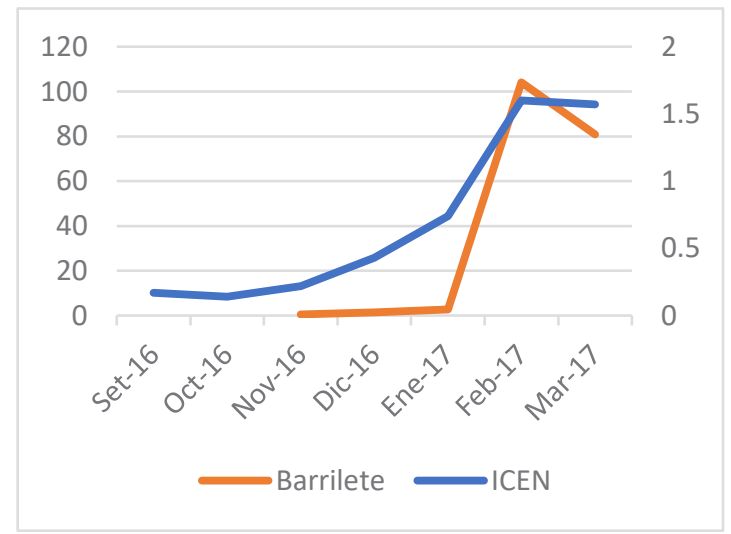

Grafico 5: Relación de las descargas de Barrilete Katsuwonus pelamis con el ICEN, de setiembre 2016 a marzo 2017

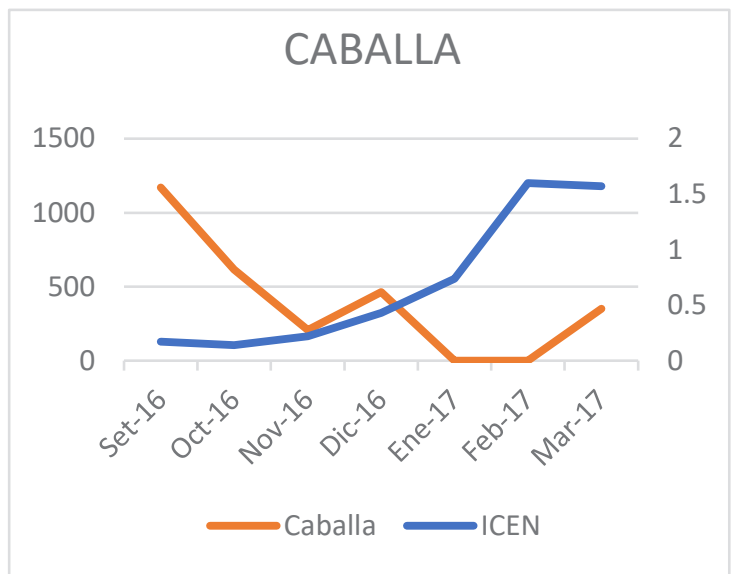

Grafico 2: Relación de las descargas de Scomber japonicus peruanus Caballa con el ICEN, de setiembre 2016 a marzo 2017

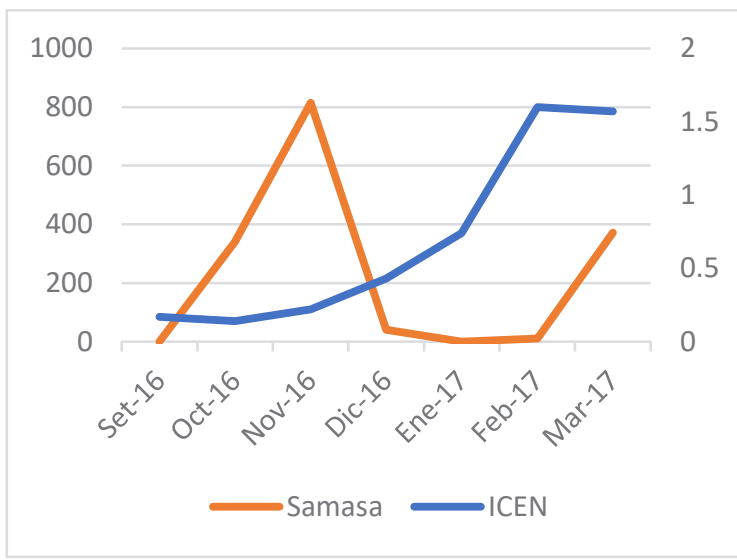

Grafico 4:Relación de las descargas de Samasa Anchoa nasus con el ICEN, de setiembre 2016 a marzo 2017

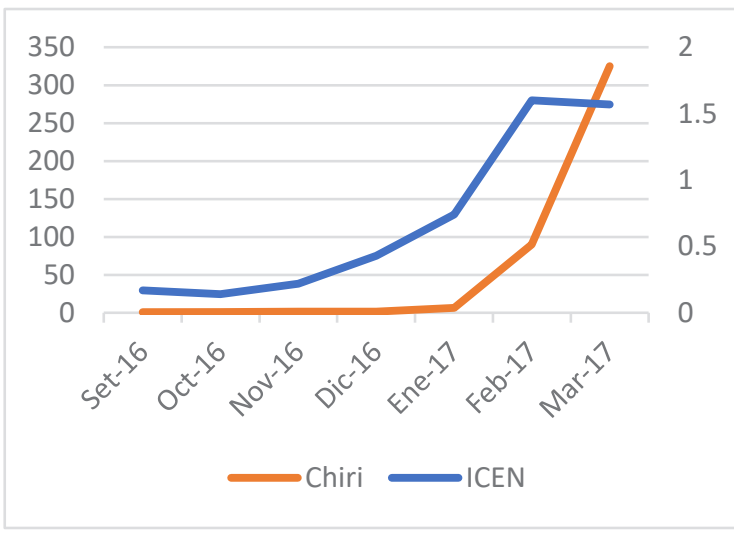

Grafico 6: Relación de las descargas de Chiri Peprilus medius con el ICEN, de setiembre 2016 a marzo 2017 
Grafico 1: Relación de las descargas de Langostino Blanco Litopenaeus vannameii con el ICEN, de setiembre 2016 a marzo 2017

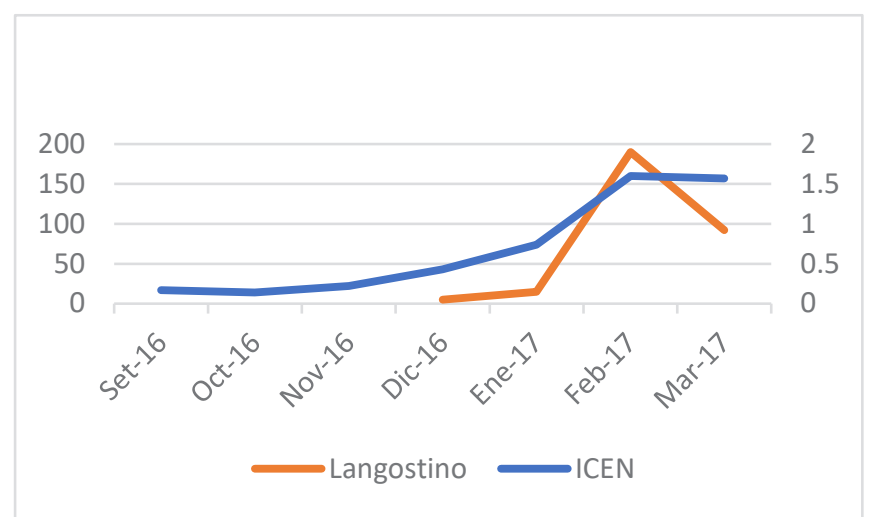

En el gráfico 1 observamos que la pota tiene dos comportamientos relativamente diferentes, en relación con el ICEN hasta el mes de diciembre, tiene un comportamiento indiferente, pero hacia enero el comportamiento es directamente proporcional, es decir a mayor valor, mayores descargas. En el gráfico 2. Notamos que para la Caballa Scomber japonicus peruanus, las descargas tienen un comportamiento inverso respecto al ICEN, lo que nos indica que disminuye a medida que el ICEN aumenta, podemos decir entonces que el Niño afecta sus descargas. En el gráfico 3. Observamos para la Cachema Cynocion analis, a pesar de estar presente durante todo el tiempo de estudios se vio afectado por la presencia del niño, lo que se refleja en el comportamiento de sus descargas, las cuales son inversas también respecto al ICEN. En el gráfico 4, para la Samasa Anchoa nasus, las mayores descargas se observan para valores bajos de ICEN, es decir tienen un comportamiento también inverso, pero se debe destacar que cuando el valor ICEN supera el valor 1, los volúmenes de descarga empiezan a disminuir, esto debido a que es una especie que si bien es cierto era indicador de aguas calientes, ha cambiado su comportamiento respecto a la TSM. En el gráfico 5, observamos para el Barrilete Katsuwonus pelamis, las mayores descargas se observan para valores altos del ICEN, es decir tienen un comportamiento también directo, llegando a reportarse mayores descargas cuando el ICEN es alto, generalmente mayor a 1. Cabe hacer mención que las descargas en su mayoría fueron en Parachique. En el gráfico 7, para el Chiri Peprilus medius, las mayores descargas se observan para valores altos del ICEN, al igual que el barrilete, es decir tiene un comportamiento también directo, llegando a reportarse mayores descargas cuando el ICEN es alto, generalmente mayor a 1. Cabe hacer mención que las descargas en su mayoría fueron en Talara.En el gráfico 7, observamos la relación entre el ICEN y las descargas de Langostino Blanco Litopenaeus vannameii, las mayores descargas se observan para valores altos del ICEN, al igual que el barrilete y el chiri, es decir tienen un comportamiento también directo, llegando a reportarse mayores descargas cuando el ICEN es alto, generalmente mayor a 1. Cabe hacer mención que las descargas en su mayoría fueron en Paita.

\section{DISCUSIÓN}

El comportamiento de la pota Dosidicus gigas, en los meses de setiembre a diciembre se comportan indiferente al ICEN, es decir no se ven afectadas las descargas, pero durante los meses de enero a marzo, su comportamiento en cuanto a descargas es positivo es decir aumentan las descargas de pota, Lo que difiere con lo encontrado por Yamashiro que encontró que los eventos El Niño pueden afectar la disponibilidad y abundancia del calamar gigante dependiendo de la intensidad, duración y cobertura de los mismos. En nuestro estudio se estableció también que si bien la pota logró mantenerse en la zona uno de los factores importantes fue su distribución vertical, en el sentido que se reportó la pesca a 150 y $200 \mathrm{~m}$ de profundidad, y por otro lado el desplazamiento horizontal hacia la zona sur. La Caballa Scomber japonicus peruanus, es una especie pelágica, que se caracteriza por ser de aguas frías, 
encontramos que se ven afectadas sus descargas a medida que las temperaturas van aumentando, y cuando el ICEN alcanza un valor por encima de 0,6; esta especie migra en busca de aguas frias, hacia el sur como lo confirman la descargas en Chimbote.

La cachema Cynosion analis, es una especie demersal, que es afectada en sus volúmenes de descarga figura 4, así observamos que los primeros meses entre setiembre y diciembre la capturas eran altas, pero en los meses de enero a marzo esta especie disminuye en sus capturas, esto se debe básicamente a que la especie trata de ubicar un estrato adecuando para poder sobrevivir, profundizándose. Esto coincide con lo encontrado por (Tresierra-Aguilar et al., 1999b), donde. En el segundo semestre de 1998 (1998B), las condiciones ambientales tendieron a la normalidad y las especies tradicionales, como Cynoscion analis, caballa, cabinza y lorna, volvieron a sus volúmenes de desembarque habituales.

Las descargas de la Samasa Anchoa nasus, una especie que era considerada de aguas calientes, que aparece en los meses de octubre, noviembre y diciembre, pero que en los meses de enero a marzo 2017, sus volúmenes disminuyen. Esto indica que si bien es cierto esta especie es considerada de aguas calientes, no es tolerable a anomalías altas de temperatura por lo que su distribución se observó con descargas más al sur. Las descargas de Barrilete Katsuwonus pelamis las cuales empiezan a tener importancia a partir de noviembre del 2016, lo cual nos da indica la presencia de especies de Aguas oceánicas tropicales, aumentando su captura hacia los meses de enero a marzo. Lo que coincide por lo encontrado con (Tresierra-Aguilar et al., 1999b), en el primer trimestre de 1998, las mayores temperaturas alcanzadas por el evento "El Niño", tuvo una clara manifestación en la presencia de especies de aguas tropicales en la pesca, como el Pez Sierra Scomberomorus maculatus, el Barrilete Katsuwonus pelamis, dorados, atunes, tiburones y Langostino Penaeus sp. El chiri Peprilus medius, especie de aguas calientes, que se acerca a la costa norte, especialmente frente a Talara, como se aprecia en Gráfico 7, el chiri es una especie de la cual se registraron descargas durante toda la época de estudios, pero sus mayores valores se alcanzan en los meses de verano, con presencia de temperaturas altas y valores ICEN altos. El langostino Litopenaeus vannamei, es una especie que aparece cuando la TSM aumenta y sobre todo con la afluencia de aguas dulces, producto de la entrada del rio Chira en Paita, El langostino es una especie que empieza a tener reportes de descargas a fines del mes de diciembre y luego se incrementa la descarga durante los meses de verano, en el último mes la descarga disminuye, debido a que se prohibió el uso de la red de arrastre que es el arte de pesca que usan las embarcaciones para su captura.

\section{CONCLUSIONES}

El Evento el Niño trae consigo, desastres naturales, baja producción, pero nos da la oportunidad de aprovechar recursos nuevos, asi en la pesca podemos indicar que las pesquerías de Barrilete, Chiri y Langostino son las más importantes durante un Evento El niño

La especie Barrilete, es una especie que muy bien puede ser aprovechada por la industria pesquera en la elaboración de conservas de pescado.

La especie chiri, es una especie ocasional, que se podría ser muy aprovechas, para la exportación como producto congelado, lo que supliría en parte los problemas de las especies tradicionales

El langostino es otro recurso que aparece en cada Evento El Niño, como se aprecia en diciembre cuando ya se observa las descargas de este recurso. Para poder capturar este recurso los pescadores utilizan una red de Arrastre, arte de pesca que está prohibido utilizar dentro de las 5 millas área donde se desenvuelve este recurso. Por ello se debe plantea algunas medidas que posiblemente permitan la pesca de este recurso, pero con un plan de ordenamiento adecuado, y que permita el aprovechamiento del recurso en el sentido que al no ser pescados, este recurso se perderá 


\section{REFERENCIAS BIBLIOGRÁFICAS}

Adams, G. D., \& Flores, D. (2016a). Influencia de El Niño Oscilación del Sur en la disponibilidad y abundancia de recursos hidrobiológicos de la pesca artesanal en Ica, Perú. Revista de biología marina y oceanografía, 51(2), 265-272.

Adams, G. D., \& Flores, D. (2016b). Influencia de El Niño Oscilación del Sur en la disponibilidad y abundancia de recursos hidrobiológicos de la pesca artesanal en Ica, Perú. Revista de biología marina y oceanografía, 51(2), 265-272.

Espino Sánchez, M., \& Yamashiro Guinoza, C. (1996). El Niño y la ordenación pesquera en el Perú.

Espinoza, J. (1996). El Niño y sus implicaciones sobre el medio ambiente.

Galarza, E., Zegarra, K., \& Noelia, J. (2015). Pesca artesanal: Oportunidades para el desarrollo regional. Universidad del Pacífico.

Tresierra-Aguilar, A., Culquichicón, Z., \& Veneros-Urbina, B. (1999a). Efecto del fenómeno" El Niño 1997 -98" en el desembarque de los recursos pesqueros artesanales de puerto Salaverry (Trujillo). Revista peruana de Biología, 6(3), 160-163.

Tresierra-Aguilar, A., Culquichicón, Z., \& Veneros-Urbina, B. (1999b). Efecto del fenómeno" El Niño 1997• 98" en el desembarque de los recursos pesqueros artesanales de puerto Salaverry (Trujillo). Revista Peruana de Biología, 6(3), 160-163.

Valdivia, E., \& Arntz, W. E. (1985). Cambios en los Recursos Costeros y su Incidencia en la Pesquería Artesanal durante «El Niño» 1982-1983.

Yamashiro Guinoza, C., Marín Soto, W., \& Argüelles Torres, J. (2016). El recurso calamar gigante en la costa peruana y El Niño. 